\title{
MDCT Findings in Focal Fat Infarction of Appendage of Falciform Ligament- An Unusual Cause of Epigastric Pain
}

\author{
Ranjana Guptaํㅜ, Rishabh Yadav², Simar Abheet Singh³, Puneet Mittal4, Narender Yadav ${ }^{5}$
}

\begin{abstract}
${ }^{1}$ Department of Radiodiagnosis, Maharishi Markandeshwar Institute of Medical Sciences and Research (MM Deemed to be University) Mullana, Ambala, Haryana, India. ${ }^{2}$ Department of Radiodiagnosis, Maharishi Markandeshwar Institute of Medical Sciences and Research (MM Deemed to be University) Mullana, Ambala, Haryana, India. ${ }^{3}$ Department of Radiodiagnosis, Maharishi Markandeshwar Institute of Medical Sciences and Research (MM Deemed to be University) Mullana, Ambala, Haryana, India. ${ }^{4}$ Department of Radiodiagnosis, Maharishi Markandeshwar Institute of Medical Sciences and Research (MM Deemed to be University) Mullana, Ambala, Haryana, India. ${ }^{5}$ Department of Radiodiagnosis, Maharishi Markandeshwar Institute of Medical Sciences and Research (MM Deemed to be University) Mullana, Ambala, Haryana, India.
\end{abstract}

\section{INTRODUCTION}

The pathophysiology and clinical course of focal fat infarction of appendage of falciform ligament is similar to other acute fat infarction abdominal conditions like omental infarction and epiploic appendagitis in that it presents with acute abdominal pain and responds well to conservative treatment. Importance of radiological diagnosis is to avoid unnecessary surgery.[1] Torsion and focal fat infarction of appendage of falciform ligament is an extremely rare condition which presents with acute abdomen. It can mimic other serious abdominal conditions which require urgent surgical intervention. It is important to be aware of its computed tomography (CT) findings so as to avoid unnecessary surgical intervention. We present CT findings in case of focal fat infarction of appendage of falciform ligament in a young male who presented with acute epigastric pain.

\section{PRESENTATION OF CASE}

A 25-year-old male patient presented with acute onset of epigastric pain, tenderness and guarding. It was accompanied by nausea and vomiting. Abdominal ultrasound examination was normal with no evidence of gall bladder disease. Serum amylase and lipase levels were within normal limits. Multi-detector computed tomography (MDCT) scan was performed for a definitive diagnosis. It showed a well-demarcated focal fatty inflammatory mass just beneath anterior abdominal wall in the region of falciform ligament in extra-peritoneal location. It was extending anterior and inferior to the left lobe of liver causing its mild smooth indentation (figure 1). Based on the typical CT appearance, diagnosis of torsion with focal fat infarction of appendage of falciform ligament was made. Patient was managed conservatively with analgesics. Patient's symptoms improved and he was almost symptom free after two weeks. No exploratory laparotomy was performed.
Corresponding Author:

Rishabh Yadav,

Department of Radiodiagnosis, Maharishi Markandeshwar Institute of Medical Sciences and Research, Mullana, Ambala, Haryana, India. E-mail: drrishabh.always@gmail.com

DOI: $10.14260 /$ jemds/2020/84

Financial or Other Competing Interests: None.

How to Cite This Article:

Gupta R, Yadav R, Singh S, et al. MDCT findings in focal fat infarction of appendage of falciform ligament- an unusual cause of epigastric pain. J. Evolution Med. Dent. Sci. 2020;9(06):370-371, DOI:
Submission 29-11-2019,

Peer Review 17-01-2020,

Acceptance 24-01-2020,

Published 10-02-2020. 


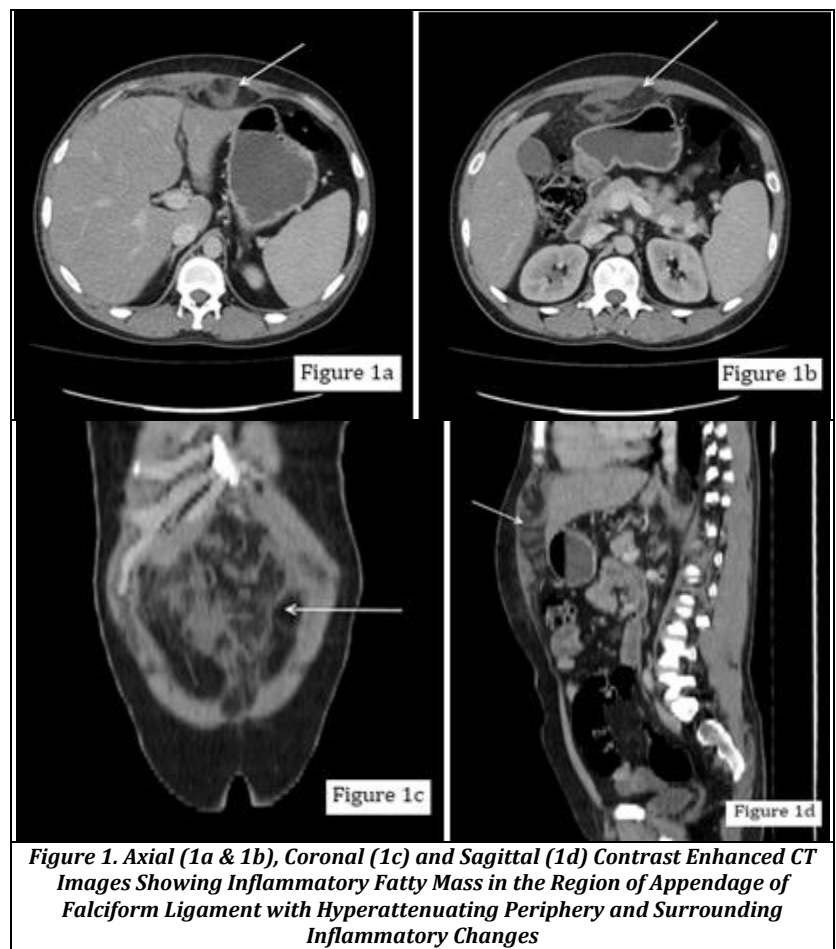

DISCUSSION

Falciform ligament is a double fold of peritoneum which is attaches liver to the anterior abdominal wall. It separates medial and lateral segments of left lobe. Ligamentum teres is contained within its free edge. It contains variable amount of loose fatty tissue.[1,2] Pathologies of falciform ligament are rarely encountered in clinical practice and include tumours, gangrene secondary to pancreatitis, internal hernias, lipomas and torsion. ${ }^{[1]}$

Torsion and infarction of appendage of falciform ligament is and extremely rare condition that is usually diagnosed on surgery. With increasing using of imaging studies, it has been occasionally reported on CT and sonography with only less than 20 cases reported described on imaging reports. ${ }^{[3,4]}$ Its pathophysiology is similar to torsion of greater omentum and epiploic appendages. All these fatty tissues have only fragile blood supply, so once there is torsion, its rapidly leads to thrombosis of their draining vein which results in haemorrhagic infarction. The thrombosed vein may sometimes be recognised as hyperattenuating dot in the centre of these lesions. [3] Despite acute presentation, these conditions are usually self-limiting and respond well to conservative treatment.

It can be diagnosed on both sonography and CT. Sonography findings show fatty echogenic inflammatory mass beneath abdominal wall in the region of falciform ligament at the site of tenderness. Sonography, by virtue of its real time nature, can help ascertain extra-peritoneal nature of the lesion as does not move with respiration. [5] CT can definitely pinpoint the exact location of abnormality to the falciform ligament. The inflamed mass may show peripheral hyperattenuating rim and central dot of thrombosed venous channel. [2-5]

\section{CONCLUSIONS}

Torsion and focal fat infarction of appendage of falciform ligament is an extremely rare condition that presents with acute epigastric pain Awareness of typical radiological findings can help in non-invasive diagnosis of this condition and thereby prevents unnecessary surgery.

\section{REFERENCES}

[1] Maccallum C, Eaton S, Chubb D, et al. Torsion of fatty appendage of falciform ligament: acute abdomen in a child. Case Rep Radiol 2015;2015:293491.

[2] Li XP, Xu DC, Tan HY, et al. Anatomical study on the morphology and blood supply of the falciform ligament and its clinical significance. Surg Radiol Anat 2004;26(2):106-9.

[3] Vanderschueren L, Coulier B. Focal fat infarction of the falciform ligament: typical CT appearance of a rare form of intra-peritoneal focal fatty infarction. J Belg Soc Radiol 2019;103(1):28.

[4] Indiran V, Dixit R, Maduraimuthu P. Unusual cause of epigastric pain: intra-abdominal focal fat infarction involving appendage of falciform ligament - case report and review of literature. GE Port J Gastroenterol 2018;25(4):179-83.

[5] Lloyd T. Primary torsion of the falciform ligament: computed tomography and ultrasound findings. Australas Radiol 2006;50(3):252-4. 